\title{
SOBRE LAS FUNCIONES DE PUES EN POSICIÓN FINAL: UN ANÁLISIS A PARTIR DEL SISTEMA DE UNIDADES DEL GRUPO VAL.ES.CO.
}

\author{
Josaphat Enrique GuiLlén EscamiLla \\ Escuela Nacional de Antropología e Historia (México)
}

\section{RESUMEN}

Uno de los marcadores discursivos más comunes en español es pues. De manera general, se ha descrito que su diversidad funcional está asociada a la gran libertad que tiene para aparecer en prácticamente cualquier posición. No obstante, es común encontrar estudios que adoptan una postura laxa en cuanto a esta noción, por lo que no es del todo claro a qué se refieren con posición inicial, media y final. Por otra parte, los estudios sobre las funciones de pues en posición final son escasos en comparación con las otras dos posiciones. Por estos motivos, el presente estudio tiene como objetivo analizar las funciones de pues en posición final a partir del sistema de unidades propuesto por el Grupo Val.Es.Co. (2014, Pons 2016). Los datos para nuestro estudio provienen de las 108 entrevistas que componen el Corpus Sociolingüístico de la Ciudad de México (Martín Butragueño y Lastra 2011, 2013, 2015). El análisis de los datos permitió identificar que el marcador pues puede aparecer en posición final de intervención, acto y subacto, por lo que desarrolla cuatro funciones: (i) reformulación, (ii) generador de inferencias, (iii) modalización y (iv) regulación; todas ellas descritas con anterioridad, aunque no todas en posición final. En resumen, se confirma que, al adoptar una postura más restrictiva de posición y al considerar la unidad en la que aparece el marcador, se pueden identificar ciertos matices funcionales que contribuyen a llevar a cabo una caracterización más puntual de los marcadores discursivos.

PALABRAS CLAVE: marcadores discursivos; discurso; Val.Es.Co.; segmentación del discurso; pragmática.

\section{AbSTRACT}

Pues is one of the most common discourse markers in Spanish. In general terms, it has been stated that its functional diversity is mainly due to its versality to appear virtually anywhere in discourse. However, in some studies the issue of the positioning of this discourse marker is somewhat less than clear, as no categorical definition is provided of what exactly is meant by initial, medial and final posi- 
tion. On the other hand, the studies on the functions of pues in final position are scarce in comparison with the other two positions. This paper aims thus to fill in this gap delving into the functions of pues in final position in the light of the discourse segmentation theory proposed by Grupo Val.Es.Co. (2014, Pons 2016). The data for our research comes from Corpus Sociolingüístico de la Ciudad de México (Martín Butragueño and Lastra 2011, 2013, 2015) which consists of 108 interviews. Our analysis of the data has allowed us to detect that pues can appear in the final position of an intervention, act, or subact and can accordingly be said to fulfill four functions: (i) reformulation, (ii) inference making, (iii) modalization, and (iv) regulation. All these functions have been previously described in initial and medial position, but not in final position. Finally, it is confirmed that if we adopt a restrictive view on position and we also take into account the unit in which the marker is found, it is then possible to identify certain features that contribute to a finer-grained characterization of the functions of the discourse markers.

KEYWORDS: discourse markers; discourse; Val.Es.Co.; discourse segmentation; pragmatics.

Fecha de recepción: 07/01/2020

Fecha de aceptación: 23/03/2020

Fecha de la versión definitiva: 31/03/2020

\section{INTRODUCCIÓN}

Desde la década de los noventa, el estudio de los marcadores discursivos ha crecido de manera significativa, su análisis se ha abordado desde diversos enfoques y perspectivas teóricas. En general, se ha buscado establecer clasificaciones que engloben todas las funciones que pueden desempeñar en la comunicación y su posible aplicación en diversos ámbitos de la lingüística. En este contexto, uno de los marcadores que más atención ha recibido es pues y se ha concluido que puede desempeñar múltiples funciones, lo que se ve reflejado en la gran movilidad que tiene dentro del discurso, así como en las propiedades prosódicas que lo dotan de matices funcionales. De tal forma, existen estudios que abordan su descripción general (Portolés 1989; Martín Zorraquino y Portolés 1999; Portolés 1998; Calsamiglia y Tusón 1999; Uribe 2006; Guevara 2015; Muñoz 2017, inter alia); otros más, se enfocan en su estudio dentro de variedades específicas del español, como el de Colombia (Travis 2005; Grajales 2011), La Habana (González y Perdomo 2018), Lima (Villa 2017) y México (Vázquez 2013, 2017). Asimismo, también se ha estudiado desde una perspectiva prosódica (Dorta y Domínguez 2001, 2006; Martín Butragueño 2003; Martínez y Domínguez 2006), desde una perspectiva de enseñanza de español como L2 (Domínguez 2016; Holgado 2017; Lončar y Mušura 2018; Pascual 2019) e, incluso, desde un enfoque de lingüística clínica (Guillén 2013; Mendoza y Guillén 2016; Guillén 2017). 
En este escenario, se ha destacado la alta movilidad de este marcador -puede aparecer en posición inicial, media o final- lo que se ha asociado con una alta polifuncionalidad. No obstante, en muchos de los estudios no queda claro qué se entiende por posición final por lo que, siguiendo a Pons (2007: 278), resulta necesario incorporar «una visión más restrictiva de la noción posición» para refinar las descripciones funcionales de este marcador. Evidentemente, al hacerlo también se precisa incluir una propuesta de sistema de unidades. Por estas razones, el objetivo de este trabajo es caracterizar las funciones de pues en posición final atendiendo a la unidad en la que aparece: acto, subacto o intervención. Para tal fin, se consultó la totalidad del Corpus Sociolingüístico de la Ciudad de México (Martín Butragueño y Lastra 2011, 2012, 2015), se analizaron todas las apariciones de este marcador en posición final y, para el análisis, se retomó la propuesta del Grupo Val.Es.Co. sobre unidades y posición (Briz y Pons 2010; Pons 2016). Así pues, en $\S 1$ se describen los estudios previos sobre pues en posición final, en $§ 2$ se caracterizan las nociones de posición y el sistema de unidades del Grupo Val.Es.Co., en $§ 3$ se detalla la metodología que se utilizó para el análisis realizado en $\S 4$. Finalmente, en $\S 5$ se presenta la discusión y en $§ 6$ las conclusiones.

\section{SObRE LAS FUNCIONES DE PUES EN POSICIÓN FINAL}

Este marcador es uno de los más frecuentes tanto en la conversación coloquial como en el registro formal (Domínguez 2016), pero aún sigue siendo "poco definido, [e] insuficientemente tratado» (Uribe 2006: 9). Así pues, se ha destacado que cuenta con una amplia libertad para aparecer en prácticamente cualquier posición, aunque la final es la menos preferida $^{1}$, como lo atestigua la ausencia de casos en la entrada del Diccionario de partículas discursivas del español (cf. Briz, Pons y Portolés 2008; Porroche Ballesteros 2008).

Así, existen varias caracterizaciones de las funciones de pues en posición final, pero es común que una misma función reciba distintos nombres o, en cambio, no sea del todo claro si la posición del marcador sea, en realidad, final. Por estas razones, en esta sección se presentan las descripciones más extendidas de este marcador en posición final, con la intención de establecer una clasificación general y plantear un punto de partida común para el posterior análisis.

En primer lugar, se debe destacar que la posición final de pues ha sido reportada principalmente en variedades latinoamericanas del español, por

\footnotetext{
${ }^{1}$ Sin embargo, Villa (2017) señala que en sus datos pues ocupó la posición final en el $80 \%$ de las ocasiones, aunque no es del todo claro a qué se refiere la autora con posición final.
} 
lo que se ha considerado como una marca dialectal del español de Medellín (Grajales 2011), del español de Colombia (Travis 2005), del español limeño, del español andino (Villa 2017) y del español del sureste de México (Vázquez 2017). En conjunto, en estos estudios se han identificado las siguientes funciones: exhortativo, enfatizador, refuerzo de acto ilocutivo, reformulador, comentador o confirmativo, clarificador, inferencial y marca de final de tópico. A continuación, se explica cada una de ellas.

La función exhortativa orienta al interlocutor a pensar y restablecer sus propios comentarios, por lo que viene acompañada con un matiz de ruego, petición o mandato hacia el interlocutor (Viera 2005 apud Guevara 2015). Por esta razón, Villa (2017) sostiene que la construcción típica es: imperativo + pues. En la clasificación de Grajales (2011), esta función recibe el nombre de refuerzo de acto ilocutivo, que se caracteriza porque el marcador siempre aparece pospuesto a un verbo en forma imperativa, por lo que enfatiza la intención de mandato hacia el interlocutor. Son casos como los de (1) y (2):

(1) [I está hablando sobre algunos clientes que piden cerveza y, al final, no quieren pagar]

663 I: entonces les haces algo, pues no tarda el día que va a decir: «¿sabes qué?, pues ¡vamos!»

$664 \mathrm{E}$ : claro

665 I: y no, mejor: «¿sabes qué?, pues ándenle pues, váyanse», es lo que ha, es lo que hemos hecho siempre desde que hemos estado aquí (entrevista 37 ME-049-21H-99).

(2) 599 I: ¿¿sí?, «que necesitan un chofer», «no, pues quién sabe quién, dónde», «pues búsquelo pues» (entrevista $62 \mathrm{ME}-267-23 \mathrm{H}-06$ ).

Por su parte, la función de enfatizador sirve para «llamar la atención e interés del interlocutor, e intensificar el efecto que el contenido enunciado pudiera provocar en él» (Guevara 2015: 309). En este mismo sentido, varios autores (Calsamiglia y Tusón 1999; Vázquez 2017; Villa 2017) coinciden en atribuirle valores modales y atenuantes, ya que «en la mayoría de los casos pues refuerza el sentido de la palabra que acompaña» (Uribe 2005: 566). En ejemplos como estos, el marcador ya no tiene un:

carácter conectivo [... sino] un valor modal asociado de refuerzo [por lo que] No hay reformulación aquí. No hay un primer miembro discursivo que deba ser reinterpretado en términos de un segundo miembro por la sencilla razón de que dicho miembro no existe (Pons 2007: 280).

Son casos como los de (3) y (4):

(3) $463 \mathrm{E}$ : oye y al momento de bailar, ¿no desconoces a los amigos?, por el rollo este del protagonismo pues (entrevista $42 \mathrm{ME}-271-21 \mathrm{H}-06$ ). 
(4) 286 I: mm, me sentía yo, pues, capaz pues

$287 \mathrm{E}: \mathrm{mh}$

288 I: ya ni los jefes ya nomás me mandaban: «ahí, que lo vean con él» (entrevista 66 ME-282-23H-06).

En cuanto a la función de reformulación, el marcador indica corrección, paráfrasis, recapitulación o ejemplificación (Travis 2005; Dorta y Domínguez 2011; Muñoz 2017; Villa 2017). Específicamente, este uso está asociado a una estrategia discursiva, una donde el hablante busca «to make clearer their description and terminate the problematic nature of their telling» (Vázquez 2013: 299-300). En estos casos, el marcador aparece después de que ya se ha reformulado el habla previa, por lo que no señala el fin de un tópico, sino el de una acción (Vázquez 2017). Son casos como los de (5) y (6):

(5) 244 I: o sea no no me siento capaz de eso/ total que/ éramos/ primero éramos cinco personas/ pero en sí/ el trabajo lo hacíamos cuatro mujeres/ iban dos señores que son esposos de dos seño-iban dos matrimonios pues (entrevista 70 ME-276-23M-06).

(6) $537 \mathrm{E}$ : o sea, no has pasado por cualquier cosa sí ha sido...

$538 \mathrm{I}: \mathrm{mmm}$, ya he estado en hospitales, he estado en cárceles, y ya la familia pues ya está preocupada

$539 \mathrm{E}:$ en la cárcel te refieres ¿al anexo o aparte?

540 I: en las procus

$541 \mathrm{E}:$ ¿ah sí?

542 I: en los separos pues (entrevista 77 ME-304-11H-07).

También se han reportado casos donde el marcador aparece al final de frases hechas como jándale pues!, ¡órale pues!, lo que se ha interpretado como una marca de conformidad con lo expuesto previamente (Vázquez 2017) $y$, en otros casos, como una marca dialectal por la «tendencia que tienen los hablantes de Medellín a construir enunciados con el marcador pues al final, como en rapidito pues, qué hubo pues, entre otros» (Grajales 2011: 38).

Por su parte, Travis denomina marca de final de tópico los casos donde pues «mark the utterances they follow as final, as a point that should be taken to conclude what the speaker has to say about the topic under discussion» (2005: 280) como en (7):

(7) 427 I: ... yo estaba muy feliz/ porque iba a ser universitaria/ ¿no?/ porque/ la Unam me esperaba entonces/ a partir de ese momento/ fui la $\langle\ldots\rangle$ / es mi número de cuenta de la/ de la universidad/ y feliz pues ¿no? (entrevista 24 ME-259-32M-05)

Finalmente, hay otras funciones menos atestiguadas, que Villa (2017) describe como confirmativo (corrobora el enunciado previo del interlocutor), clarificador (aparece después de información obvia -en casos como 
sí pues- (Vázquez 2017) e inferencial (el contenido se infiere del discurso precedente), aunque las primeras dos podrían incluirse en la función más general de enfatizador, gracias a sus matices modales.

En resumen, podemos partir de una clasificación general que incluye cinco funciones de pues: (i) exhortativo, (ii) enfatizador, (iii) reformulador, (iv) marca de final de tópico e (v) inferencial.

\section{SOBRE UNIDADES Y POSICIÓN}

El sistema de unidades propuesto por el Grupo Val.Es.Co. (Briz 1998, 2000, 2006; Briz y Grupo Val.Es.Co. 2002, 2003; Briz y Pons 2010; Grupo Val.Es.Co. 2014; Pons 2016) -basado en el modelo de la Escuela de Ginebra (Roulet et al. 1985, 2001)- es una herramienta de análisis que sirve, entre otras cosas, para el estudio de los marcadores discursivos. Desde esta perspectiva, se presta especial atención al tipo de unidades que conforman o del que son parte y a la posición que tienen en la conversación, ya que estos dos aspectos, en conjunto, dotan de matices funcionales a estas partículas discursivas.

Como punto de partida, en este sistema se proponen ocho unidades -discurso, diálogo, intercambio/alternancia de turnos, intervención/turno, acto y subacto-, donde la conversación tiene como límite superior el discurso, cuya unidad mínima es el intercambio/alternancia de turnos, y como límite inferior el acto y subacto, que componen la unidad máxima monológica, la intervención/turno. Así pues, la unión de dos intervenciones de distintos emisores constituye un intercambio, que puede estar formado por una intervención iniciativa -directa o indirecta- (aquella que intenta provocar o provoca habla posterior) y otra reactiva -cooperativa, evaluadora, fática o completiva- (la respuesta o reacción) o compuesta (consta de una intervención que es reactiva e iniciativa a la vez).

En este contexto, se consideran tres dimensiones que ayudan a distinguir entre tipos de unidades. La primera es la dimensión estructural, de naturaleza interna, que señala la manera en que los constituyentes se suceden para construir un mensaje. La segunda es la dimensión social, de naturaleza externa, relacionada con la influencia que ejercen los participantes sobre la estructura conversacional resultante. Por último, la dimensión informativa muestra la forma en que se divide un mensaje (Grupo Val.Es.Co. 2014). Específicamente, esta división permite diferenciar entre unidades de índole estructural y unidades de índole social, por eso la intervención goza solo de un carácter estructural interno, es decir, corresponde únicamente a la producción de material fónico por parte de un emisor; en contraste, el turno está, además, revestido de un carácter social externo, ya que influye efectivamente en el desarrollo temático de la conversación. 
Por su parte, la intervención está conformada por unidades más pequeñas llamadas actos. Estas unidades representan una acción comunicativa completa y, en consecuencia, son aislables, por lo que un acto puede constituir -por sí mismo- una intervención:

(8) 244 I: \#o sea, lo de los peseros empezaron siendo coches\#

$245 \mathrm{E}$ : \#sí\# \#sí sabía esa historia de que eran taxis colectivos, ¿no?\# (entrevista 24 ME-259-32M-05)

Así, en (8) la intervención 244 I consta de un solo acto, mientras que la intervención 245 E se conforma de dos, donde cada uno de ellos, de manera aislada, puede constituirse en una intervención reactiva, como se muestra en $(9 a)$ y $(9 b)$ :

(9a) 244 I: \#o sea, lo de los peseros empezaron siendo coches\# $245 \mathrm{E}$ : \#sí\#

(9b) $244 \mathrm{I}:$ \#o sea, lo de los peseros empezaron siendo coches\# $245 \mathrm{E}$ : \#sí sabía esa historia de que eran taxis colectivos, ¿no?\#

Finalmente, los subactos son las unidades informativas mínimas en las que se puede dividir un acto y, según su valor, pueden ser clasificados en: (i) subactos sustantivos directores (SSD, núcleo proposicional del acto y portador de la fuerza ilocutiva), (ii) subactos sustantivos subordinados (sss, dependientes semántica e informativamente de los SSD), (iii) subactos subordinados topicalizados (SSTop, constituidos por elementos desplazados a la derecha o a la izquierda por ser informativamente más relevantes), (iv) subactos adyacentes textuales (SAT, organizan y distribuyen el flujo del habla), (v) subactos adyacentes modalizadores (SAM, señalan algún tipo de matización, sea una atenuación o una intensificación), y (vi) subactos adyacentes interpersonales (SAI, señalan la interacción entre hablante y oyente) (Pons 2008; Briz y Pons 2010; Grupo Val.Es.Co. 2014).

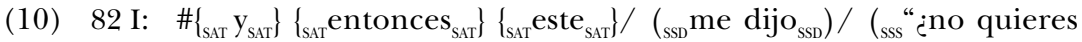

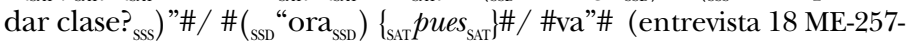
$32 \mathrm{H}-05)$.

En (10), la intervención está formada por tres actos, el primero constituido por tres subactos adyacentes textuales, un subacto sustantivo directivo y un subacto sustantivo subordinado; mientras que el segundo contiene un subacto sustantivo directivo y un subacto adyacente textual, y el último es un acto per se.

Así pues, con estas especificaciones, se refinan las nociones de posición inicial, intermedia y final, pues ahora resultará más exacto decir posición inicial de diálogo, posición inicial de intervención, posición inicial de acto o posición inicial de subacto. Lo mismo se aplica para el resto de 
las posiciones. En resumen, si se considera tanto el tipo de unidad como la posición, el análisis de las funciones de los marcadores discursivos será más detallado, ya que ambos aspectos agregan matices funcionales a estos elementos. Así, en el caso particular de esta investigación, la posición relevante será la final por lo que en nuestro análisis nos centraremos en la unidad en la que aparece el marcador.

\section{Metodología}

Para este estudio se empleó el Corpus Sociolingüístico de la Ciudad de México (CSCM, Martín Butragueño y Lastra 2011, 2012, 2015). En total, el CSCM incluye 108 entrevistas organizadas en tres niveles de instrucción ${ }^{2}$ : alto (36), medio (36) y bajo (36). Cada nivel está subdivido, a su vez, en tres grupos etarios: jóvenes (20 a 34), adultos (35 a 54) y mayores (55 en adelante). Por último, los informantes están agrupados por género. En cuanto al contenido de las entrevistas, los temas tratados incluyen el trabajo, la familia, el tiempo libre, entre otros, así que los tópicos fueron similares en todos los informantes.

Ahora bien, debemos señalar que este no es un estudio de corte sociolingüístico, pero se decidió emplear este corpus por el estilo empleado en las entrevistas, que se encuentra entre los polos de la entrevista semiinformal [+formal] y la conversación grabada [-formal], que llega a mostrar pocas diferencias con respecto a la conversación coloquial entre conocidos. En este sentido, los autores señalan que era común que algunas entrevistas comenzaran presentando una estructura semi-informal, pero -en el transcurso de la interacción- se desarrollaban como conversaciones.

En concreto, se revisaron las transcripciones de las 108 entrevistas y se rastrearon todas las apariciones de pues en posición final de intervención, acto o subacto, incluyendo las variantes con reducción fónica pos, pus y ps. Posteriormente, se procedió con el análisis de las funciones del marcador, considerando principalmente la unidad en la que aparecía. En este sentido, cabe mencionar que aquellos casos donde pues aparecía al final de un acto no concluido -como cuando el interlocutor arrebata el turno o el hablante tiene problemas con la formulación del turno- no fueron considerados

${ }^{2}$ El nivel más bajo incluye a las personas que han llegado a concluir la primaria, de tal forma que recibieron hasta 6 años de escolarización. En este nivel también están incluidos los analfabetos y quienes no terminaron la primaria. El nivel medio se compone por las personas que cursaron hasta la enseñanza media, o sea, personas que recibieron hasta 12 años de escolaridad. Finalmente, el nivel alto incluye a hablantes con enseñanza superior, es decir, recibieron al menos 16 años de escolaridad. Este grupo puede incorporar informantes con estudios de posgrado. 
como posición final. Finalmente, para la inclusión de los ejemplos dentro del texto se empleó el sistema propuesto en Pons (2016) para la división de la conversación en actos y subactos, excepto en los casos de final de intervención, ya que la posición es bastante transparente. Al final de cada ejemplo se incluye el número y la clave de la entrevista a la que pertenece.

\section{ANÁlisis}

Antes de iniciar, cabe destacar que la posición final no es la preferida de este marcador, ya que está ausente en 49 de las 108 entrevistas, resultado que contrasta con las otras posiciones -inicial y media- que aparecen sistemáticamente en todas las entrevistas. Por otra parte, a partir de nuestro análisis, las funciones de pues -descritas en $§ 1$ - se reagruparon de la siguiente forma: la reformulación y el generador de inferencias se mantienen igual, las funciones exhortativa y enfatizadora corresponden a la modalización y la marca de final de tópico cumple con la función reguladora. Así pues, en adelante nos detendremos a detallar estas funciones de pues en posición final.

\subsection{Reformulador}

En nuestros datos, la función de pues como reformulador fue la más común. En estos casos, el marcador indica la corrección de un elemento léxico o una secuencia discursiva, esto es, la reformulación se da a partir de un "proceso retroactivo que permite explicar, rectificar, reconsiderar, recapitular o separarse de la formulación anterior» (Garcés 2008: 69). De tal forma, encontramos casos donde la reformulación se presenta cuando el hablante se percata de que no ha sido capaz de contribuir efectivamente con la conversación, como en (11):

(11) [Hablando de cuándo I conoció a su esposa]

$90 \mathrm{E}:$ y luego/ eh mm/ ¿cuándo vas a Acapulco? (risa)

91 I: ¿cuándo voy a Acapulco?

$92 \mathrm{E}$ : ¿cuándo llegaste a Acapulco?/ bueno/ a... (silencio)

93 I: ¿cuándo llegué a Acapulco?

$94 \mathrm{E}$ : ¿cuándo conociste a tu mujer/ pues?

95 I: no/ ya la conocí en// en noviembre/ en un veinte de noviembre del noventa y quién sabe cuántos (silencio) (entrevista 51 ME-26922H-06).

En (11), E supone que cuando I fue a Acapulco conoció a su esposa, por eso cree que con preguntar sobre ese viaje es suficiente para que I interprete qué quiere saber en realidad. No obstante, la suposición de E es 
errónea e I no comprende las preguntas, lo que está reflejado en los turnos 91 y 93, cuando las repite. Es hasta el turno 94 cuando E se da cuenta que no está siendo claro y reformula la pregunta, haciéndola explícita.

En otros casos, la reformulación se establece entre elementos léxicos, con la intención de hacer más preciso el significado de una palabra o, incluso, para presentar una descripción de una determinada palabra, como en (12):

(12) 56 I: $\quad \#\left\{_{S A T} y_{S A T}\right\}\left(_{S S D}\right.$ un barrio se componía de/ $\left\{{ }_{S A T} p u_{e s}\right\}$ de puestos/ jaca-

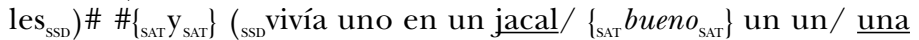
casa hecha de madera ${ }_{\mathrm{SSD}}$ ) $\left\{_{\mathrm{SAT}}\right.$ pues $\left._{\mathrm{SAT}}\right\} /\left\{_{\mathrm{sal}} \mathrm{n} \mathrm{no} ?_{\mathrm{SAI}}\right\} \#$ (entrevista $102 \mathrm{ME}-$ 314-13H-07).

En (12), I está narrando cómo eran los barrios en su infancia y menciona que vivían en jacales, pero se percata de que es posible que el interlocutor no sepa a qué se refiere con eso, por lo que reformula e introduce una descripción: una casa hecha de madera. Esta precisión comienza desde la aparición de bueno, que también cumple con funciones de reformulación, y concluye con la posposición de pues.

En otras ocasiones, los hablantes tienen problemas para construir el turno porque se dan cuenta de que lo que iban a decir puede ser socialmente censurable, así que -después de un titubeo- matizan y reformulan, como en (13):

(13) 379 I: ¿no estoy ubicando mis ideas?

$380 \mathrm{E}$ : (risa) \# $\left\{_{\mathrm{SAM}} \mathrm{no}_{\mathrm{SAM}}\right\} / / /\left\{_{\mathrm{SAM}} \mathrm{no}_{\mathrm{SAM}}\right\}\left\{_{\mathrm{SAT}} \mathrm{es} \mathrm{que}_{\mathrm{SAT}}\right\}\left(_{\mathrm{SSD}} \mathrm{sí}\right.$ me quedo pensando como en/ en México/ $\left\{{ }_{\text {sar }}\right.$ no? $\left.{ }_{\text {SAI }}\right\}$ siempre los morenos son como/ una de $\left.\operatorname{dos}_{\mathrm{sSD}}\right) /\left\{_{\mathrm{sAl}} \mathrm{ino} ?_{\mathrm{sAl}}\right\} /$ (sss $_{\mathrm{o}}$ muy amados o muy/ o no no/ $\underline{\text { no }}$

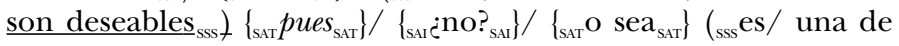
$\mathrm{dos} /$ nunca pueden ser neutrales $_{\mathrm{sss}}$ )\# (entrevista $\left.48 \mathrm{ME}-265-21 \mathrm{M}-06\right)$.

En (13), E menciona que los morenos solo tienen dos opciones: ser muy amados y, se podría esperar, muy odiados, pero se da cuenta de lo fuerte que podría ser esa afirmación por lo que matiza y reformula diciendo que no son deseables. Esto queda más claro si atendemos a la pausa y los titubeos que siguen a o muy. Así pues, lo que tienen en común todos estos casos es que regularmente se presentan titubeos, falsos inicios y/o pausas antes de la aparición de la reformulación, así como la posposición de pues a dicha corrección.

\subsection{Generador de inferencias}

La segunda función es lo que se ha llamado generador o activador de inferencias, donde lo que señala pues es el proceso inferencial que debe 
seguir el interlocutor. En otras palabras, indica la conclusión a la que tiene que llegar el oyente a partir de lo comunicado previamente por el hablante. Este valor proviene del significado instruccional (Leonetti y Escandell 2012) de este tipo de marcadores y, en el caso de pues, ha sido descrito por Fuentes (2009: 291). Así, con esta función, el marcador tiene una posición final relativa, es decir, estructuralmente ocupa la posición final de intervención, pero hay contenido elidido que es el que el interlocutor debe recuperar para lograr la conclusión intentada por el hablante. De (14) a (16) se presentan ejemplos de esta función:

(14) [hablando sobre las complicaciones durante un parto]

275 I: entonces se había ahorcado/ que también los doctores/ tuvieron parte de la culpa porque

$276 \mathrm{E}:$ no habían revisado

277 I: ajá

$278 \mathrm{E}$ : cuando ella se sentía mal/ ¿no?

279 I: ella se sintió mal dos veces/ y las dos veces la regresaron// entonces pus [sí tuvieron culpa]

$280 \mathrm{E}$ : sí/ hay una negligencia médica en el país/ tremenda (entrevista 44 ME-123-21M-01).

En la intervención 275, I menciona que los médicos tuvieron parte de la culpa de lo ocurrido durante el parto, y en 279 apunta que la paciente acudió dos veces con ellos y las dos veces no le dijeron nada, con lo que refuerza lo dicho en 275. Así, el marcador genera la conclusión: los médicos tuvieron culpa, tal es así que, en 280, E destaca la negligencia médica que existe en el país.

(15) [hablando sobre el cuidado de las hijas de I]

$103 \mathrm{E}$ : ¿y no tenía alguien que le ayudara? / su mamá

$104 \mathrm{I}$ : a veces me ayudaba una persona pero/ se las llevaba yo a mi mamá

$105 \mathrm{E}$ : pues sí

$106 \mathrm{I}:$ [para que me las cuidara]

$107 \mathrm{E}:[<\ldots>]$

$108 \mathrm{I}$ : y como iba yo en la vespertina para poder cuidar a las niñas en la mañana/ y en la noche llegar a acostarlas/iba yo de dos a siete/ era un turno de la prepa

$109 \mathrm{E}$ : más o menos $<\ldots>$

$110 \mathrm{I}: \mathrm{mh} / \mathrm{y}$ B todo el día trabajaba entonces $p s$ [tenía que pedirle ayuda a mi mamá]

$111 \mathrm{E}$ : claro/ ¿y en qué trabajaba él? (entrevista 33 ME-227-33M-03).

En (15), en 108 y 110, I explica las razones por las cuales no podía cuidar a sus hijas durante la tarde y pues guía a la conclusión tenía que pedirle ayuda a mi mamá. En este caso, la función inferencial se ve reforzada por 
el conector entonces que también sirve para introducir una consecuencia o un resultado de lo expresado en las unidades previas.

(16) [hablando sobre cómo ganarse el respeto dentro de una banda]

$471 \mathrm{E}$ : [o sea] sí eras de plano así de golpear y

472 I: mm sí o sea sí así era/ mm pues es que así como que era la/ la ley de la/ de la calle [¿no? de que]

$473 \mathrm{E}:$ [ajá]

$474 \mathrm{I}:$ si te dejas de alguien/ pues [no te van a respetar]

$475 \mathrm{E}:$ sí/ te va siempre a estar molestando y sobre lo mismo (entrevista 78 ME-305-11H-07).

En (16), I está explicando cómo es la ley de la calle, en la que no debía dejarse intimidar por nadie porque si lo hacía, no se ganaba el respeto de los demás, y esta es la conclusión generada por pues en 474. Tal es así que, en 475 , E concuerda diciendo que, si lo permite, ese alguien va siempre a estar molestando.

En resumen, con esta función, el marcador siempre aparece a final de intervención y desempeña un papel instruccional o procedimental, al señalarle al interlocutor la conclusión deseada por el hablante.

\subsection{Modalización}

La tercera función es la modalización, que aquí hemos divido en dos tipos: (i) aquella que indica acuerdo -sea falso o genuino- con lo expresado anteriormente; y (ii) aquella que enfatiza lo dicho antes del marcador, o sea, cumple con valores modales asociados con el refuerzo. De (17) a (20) se presentan ejemplos de estas funciones:

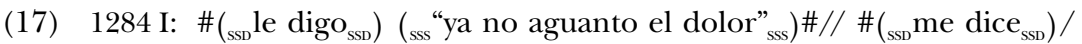
(sss "mejor espérate/ ahorita llegando a Córdoba/ te compro una

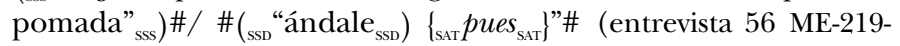
22M-02).

En (17), se presenta un diálogo entre I y su pareja, donde discuten sobre qué hacer para aliviar el dolor de I. La pareja le propone esperar hasta Córdoba para comprar una pomada, a lo que I consiente con ándale pues.

(18) $397 \mathrm{I}: \quad \#_{\left\{_{\mathrm{SAT}}\right.} \mathrm{y} \mathrm{ya_{ \textrm {SAT } } \}}$ ( $\mathrm{SSD}_{\mathrm{s}}$ según él/ ya al agacharse/ ya me quería dar un be-

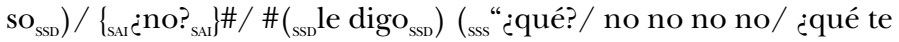

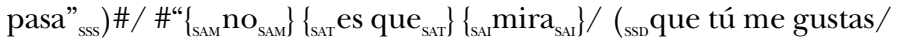
que me caes bien/ que no sé cuánto $\left.{ }_{\mathrm{ssD}}\right)$ "\# /\#( $\left({ }_{\mathrm{ssD}}\right.$ le $\left.\operatorname{dije}_{\mathrm{ssD}}\right) /$ "( sss $_{\text {no }}$

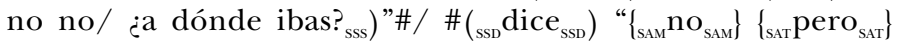

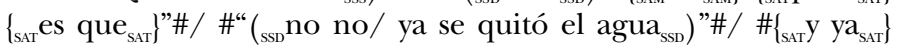




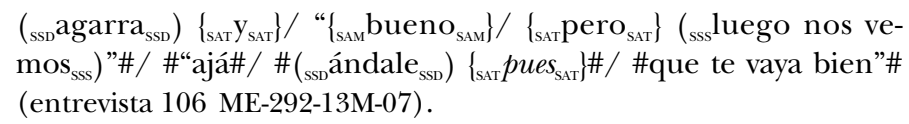

En (18), también dentro de una narración, se presenta un diálogo que tuvo I con un hombre. Luego de un momento de tensión, el hombre propone que se vean después, a lo que I accede, pero con un falso acuerdo, lo que se puede comprobar con la interjección ajá y con el segmento ándale pues.

(19) $238 \mathrm{E}:$ \#( ${ }_{\mathrm{SSD}}$ sabes qué/ que a mí sí me gustaría tener la luz $\left.\mathrm{SSD}_{\mathrm{SSD}}\right) /\left\{_{\mathrm{SAT}}\right.$ porque $\left._{\mathrm{SAT}}\right\}$

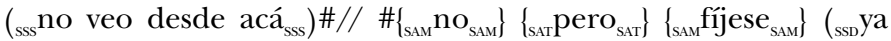
en/ hablando en serio $\left.{ }_{\mathrm{SSD}}\right)\left\{_{\mathrm{SAT}}\right.$ pues $\left._{\mathrm{SAT}}\right\} \# / \#_{\text {SAT }_{\mathrm{S}}}$ este $\left._{\mathrm{SAT}}\right\} /$ (ssD $_{\mathrm{s}}$ yo tenía

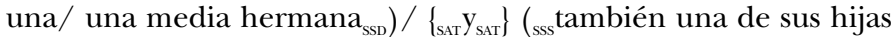
$\left\{\right.$ ssA este $\left._{\text {SAT }}\right\} /$ resultó/ que no oía $a_{\text {SSs }}$ )\# (entrevista 13 ME-006-32H-97).

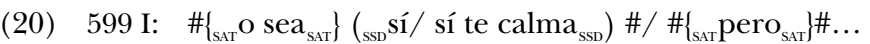

$600 \mathrm{E}$ : \#demasiado\#

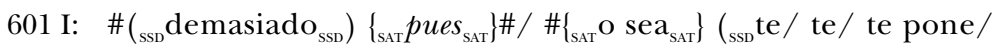
$\left\{_{\text {sAT }}\right.$ pues $\left._{\mathrm{SAT}}\right\}$ muy dormilona $\left._{\mathrm{SSD}}\right) \#$ (entrevista 55 ME-110-22M-00).

En los ejemplos (19) y (20), el marcador desempeña funciones de refuerzo, en el primer caso para indicar que se va a hablar en serio y, en el segundo, para enfatizar que el medicamento te calma demasiado. En ambos ejemplos, la posición del marcador es a final de acto.

\subsection{Función reguladora}

Finalmente, gracias a que el marcador aparece en posición final, también puede desempeñar funciones reguladoras (Pons 2000), esto es, muestra el final de la unidad discursiva en la que se inserta y tiene su ámbito de acción en el nivel de la intervención y el intercambio. Esta función podría corresponder lato sensu a lo que Travis (2005) ha llamado marca de final de tópico. Así pues, esta función se puede apreciar más claramente en los casos de pues como generador de inferencias, que siempre aparece en posición final de intervención y sirve, además, para ceder el turno, por lo que indica un Lugar de Transición Pertinente, entendido como el "punto en el que finaliza un movimiento (la unidad mínima de la conversación)» (Gallardo 1996: 66).

Hasta aquí, hemos presentado el análisis de los datos, en la siguiente sección discutimos los resultados. 


\section{Discusión}

Según vimos en $§ 2$, la función reformuladora está ampliamente reconocida. Así, en estos casos, la corrección parafrástica puede ser un resumen, una generalización o una precisión; además, el segundo miembro es comunicativamente más relevante, ya que se puede entender sin necesidad de recurrir al primer miembro. De tal forma, los elementos reformulados pueden ser elementos léxicos (se sustituye una palabra con otra), unidades discursivas completas (como en (11)), una definición o precisión como en (12), entre otros casos. En cuanto a la posición, el marcador puede aparecer a final de acto, subacto (como en (12) y (13)) e intervención (como en 11). Finalmente, cabe destacar que esta función fue la más común en el corpus.

Por su parte, la función generadora o activadora de inferencias solo ha sido mencionada anteriormente por Villa (2017). En nuestro estudio, esta función es utilizada cuando el hablante le deja al interlocutor la responsabilidad de inferir o derivar la conclusión deseada. Esta función también ha sido atestiguada en otro reformulador, o sea, que tiene un valor minimizador, esto es, el hablante «deja en suspenso la intervención para que sea el interlocutor el que extraiga la explicación o conclusiones oportunas» (Briz 2008). El ejemplo citado por el autor es:

(21) Yo no sé, la mayoría de tus preguntas, para mí, no son lógicas, o sea...

Así pues, como característica principal, con esta función el marcador debe aparecer en posición final de intervención y, gracias a su contribución con la gestión de la interacción, se constituye como un Lugar de Transición Pertinente, ya que el hablante busca una reacción del interlocutor. En consecuencia, también cumple con una función reguladora, pues señala el fin de la intervención del hablante. Finalmente, esta fue la segunda función más común en el corpus.

En cuanto a las funciones modales, también existe cierto consenso en la bibliografía, aunque principalmente se ha descrito como un elemento que añade énfasis a lo dicho previamente. En nuestro caso, encontramos que la función de modalización se puede presentar de dos maneras, una que señala acuerdo, sea genuino o falso, y otra que enfatiza la unidad que antecede al marcador. En el primer caso, se ha considerado como una marca de conformidad en frases hechas, como ¡ándale pues!, ¡órale pues!, ¡sale pues!, entre otras (Vázquez 2017); sin embargo, creemos que su empleo no se limita a ellas, ya que podemos generar nuevas frases y el matiz modal de acuerdo se mantiene: salgamos pues, vamos pues, comencemos pues, etcétera. De tal forma, consideramos que esta función está más cercana a la de 
otros marcadores con funciones modales, como claro y bueno, que indican acuerdo o desacuerdo (cf. Pons 2003). Es necesario mencionar que, igual que en el estudio de Grajales (2011), esta función se encuentra casi completamente restringida a segmentos narrativos, ya que de las 20 veces que aparece, 15 de ellas están dentro de discurso directo citado; además, es común que aparezca en frases como ándale pues y órale pues. Por último, puede aparecer a final de acto, subacto o intervención.

En cuanto a la función modal asociada con un énfasis, corresponde a la que se describió en $§ 2$, donde se explicó que «la posición final es el ámbito en el que los conectores del español desempeñan valores modales sin el concurso de otras funciones» (Pons 2007: 278). En estos ejemplos, el marcador ocupa la posición final de acto, subacto o intervención. Como en el caso de la función generadora de inferencias, el conector o sea también ha desarrollado una función parecida a esta, en particular en el español peninsular (Pons 2007), donde «puede aparecer en posición final cuando pierde su valor conector y pasa a funcionar como partícula modal» (Briz 2002: 51).

Finalmente, gracias a su posición, pues también tiene un valor secundario de regulación, es decir, muestra el final de la unidad discursiva en la que se inserta. Así pues, este valor secundario estaría relacionado con lo que Travis (2005) describió como marca de final de tópico, aunque lo que indica el marcador es el final de una unidad, principalmente actos e intervenciones.

\section{Conclusiones}

El objetivo de este trabajo era describir las funciones que desempeña pues en posición final a partir del sistema de unidades propuesto por el Grupo Val.Es.Co., principalmente porque este encuadre permite refinar la noción de posición. Así pues, de acuerdo con el análisis, se encontraron cuatro funciones: (i) reformulación, (ii) generador de inferencias, (iii) modalización -de acuerdo/desacuerdo y énfasis- y, como función secundaria asociada con la posición, (iv) regulador. Creemos que esta clasificación captura las funciones básicas de pues en posición final, además de incluir algunos matices secundarios. De tal forma, las funciones exhortativa y enfatizadora pueden englobarse en la de modalización; la marca de final de tópico correspondería a la función reguladora; mientras que la de reformulación se mantiene. En consecuencia, solo estaríamos matizando la función de generador de inferencias, ya atestiguada en Villa (2017), pero donde se concluye que pues aparece principalmente en posición intermedia de unidad de habla (p. 125) y no en posición final. 
Ahora bien, gracias al sistema de unidades empleado, se pudo determinar que, en todas las funciones, el marcador puede ocurrir en posición final de acto, subacto e intervención, excepto en la de generador de inferencias, donde está restringido a la posición final de intervención. Así, cobran más relevancia las consideraciones de Pons (2007: 278) cuando sostiene que la inclusión de una visión más restrictiva de posición final, atendiendo a unidades específicas, permite «reclasificar algunos de los usos considerados como propios de la posición intermedia» de ciertos marcadores discursivos.

Por otra parte, en el caso del español de México, se ha concluido que la posición final de pues es una marca dialectal del sureste del país (Vázquez 2017); sin embargo, también se presenta en la variante del centro, de la que provienen los datos de nuestro corpus. En este sentido, en las variantes del español americano, existe una alta homogeneidad en cuanto a las funciones de pues en posición final, por lo que probablemente no debería ser considerada como una marca dialectal.

Finalmente, queremos concluir haciendo eco a las palabras de Briz y Pons cuando sostienen que:

la variación funcional de los marcadores del discurso está limitada por su posición discursiva y por el tipo de unidad en que se integra. Ello quiere decir que [...] la unidad y la posición están en correlación en gran medida con la función de los marcadores discursivos (2010: 327). 


\section{BIBLIOGRAFÍA}

BRIZ, Antonio (1998): El español coloquial en la conversación. Esbozo de pragmagramática, Barcelona: Ariel.

- (2000): «Las unidades de la conversación», RILCE 16/2, 225-246.

- (2002): «Apuntes para la definición lexicográfica de $o$ sea». En Bernhard Poll y Franz Rainer (eds.), Vocabula et vocabularia. Études dude lexicologie et de (meta)lexicographie romanes en l'honneur D. Messner, Fráncfort: Peter Lang, 45-52.

- (2006): «Unidades del discurso, partículas discursivas y atenuantes. El caso de 'no/tienes razón'», ponencia invitada en el Congreso Discurso, interacción e identidad. Simposio Internacional en Homenaje a Lars Fant celebrado en Estocolmo (Suecia).

- (2008): «O sea». En Diccionario de partículas discursivas del español (DPDE). $<$ www.dpde.es $>$.

- y GRUPo VAL.Es.Co. (2002): Corpus de conversaciones coloquiales, Madrid: Arco/ Libros.

— y - (2003): «Un sistema de unidades para el estudio del lenguaje coloquial», Oralia 6, 7-61.

— y Salvador Pons (2010): «Unidades, marcadores y posición». En Óscar Loureda y Esperana. Acín (coords.), Los estudios sobre marcadores del discurso hoy, Madrid: Arco/Libros, 523-557.

—, Salvador Pons y José PORTOLÉs (2008): Diccionario de partículas discursivas del español (DPDE). <www.dpde.es>.

Calsamiglia, Helena y Amparo Tusón (1999): Las cosas del decir, Barcelona: Ariel.

Domínguez, Noemí (2016): «Bueno, pues, es que... en fin: ¿Qué marcadores discursivos enseñar?», Revista Signos. Estudios de Lingüistica 49(90), 3-24. (DOI: 10.4067/S0718-09342016000100001).

DorTa, Josefa y Noemí Domínguez (2001): «Polifuncionalidad discursiva y comportamiento prosódico prototípico del marcador pues», Español Actual 75, $45-53$.

— y - (2006): «La prosodia del marcador discursivo pues». En Manuel Casado, Ramón González y María Victoria Romero (eds.), Análisis del discurso: lengua, cultura, valores. Actas del I Congreso Internacional, vol. II, Madrid: Arco/Libros, 1269-1282.

Fuentes, Catalina (2009): Diccionario de conectores y operadores del español, Madrid: Arco/Libros.

GALlaRDO, Beatriz (1996): Análisis conversacional y pragmática del receptor, Valencia: Episteme.

GARCÉs, M. ${ }^{a}$ Pilar (2008): La organización del discurso: marcadores de ordenación y de formulación, España: Iberoamericana Vervuert.

GonzÁlez, Ana María y Marialys Perdomo (2018): «Aproximación al estudio de los marcadores discursivos en muestras de habla culta de La Habana», Revista de la Universidad de La Habana 285, 25-49. 
Grajales, Robinson (2011): «Funciones del marcador discursivo pues en el habla de Medellín, Colombia», Forma y Función 24, 25-45.

GRUPO VAl.Es.Co. (2014): «Las unidades del discurso oral», Estudios de Lingüística del Español 35, 13-73.

Guevara, Gloriella (2015): «Funciones del marcador discursivo pues: en un corpus oral», Dialógica: Revista Multidisciplinaria Dialógica 12, 294-323.

GuILLÉN, Josaphat (2013): El uso de marcadores discursivos y las funciones que desempeñan dentro de las producciones lingüisticas de pacientes con afasia de Wernicke: Un estudio contrastivo, Tesis de doctorado, México: El Colegio de México.

- (2017): «El uso de marcadores discursivos en la producción lingüística de un paciente con afasia motora aferente», Revista Chilena de Fonoaudiología 16, 1-16 (DOI: 10.5354/0719-4692.2017.47560).

Holgado, Anais (2017): Diccionario de marcadores discursivos para estudiantes de español como segunda lengua, Nueva York: Peter Lang.

LEONETTI, Manuel y Victoria ESCANDELL (2012): «El significado procedimental: rutas hacia una idea». En M. ${ }^{a}$ del Carmen Horno y José Luis Mendívil (eds.), La sabiduría de Mnemósine. Ensayos de historia de la lingüistica ofrecidos a José Francisco Val Álvaro, Zaragoza: Prensas Universitarias de Zaragoza, 157-167.

LONČAR, Ivana y Josipa MUŠURA (2018): «Combinatoria del marcador discursivo pues y sus correspondencias en la lengua croata», Pragmalingüistica 26, 186-198 (DOI: http://dx.doi.org/10.25267/Pragmalinguistica.2018.i26.09).

Martín ButragueÑo, Pedro (2003): «Hacia una descripción prosódica de los marcadores discursivos. Datos del español de México». En Pedro Martín Butragueño y Esther Herrera (comps.), La tonía, dimensiones fonéticas y fonológicas, El Colegio de México: México, 375-402.

- y Yolanda Lastra (coords.) (2011): Corpus sociolingǘstico de la ciudad de México, vol. I: Hablantes de instrucción alta, México: El Colegio de México. $<$ http://lef. colmex.mx/index.php/investigaciones/corpus-sociolingueistico-de-la-ciudadde-mexico-cscm>.

- y - (coords.) (2012): Corpus sociolingüistico de la ciudad de México, vol. II: Hablantes de instrucción media, México: El Colegio de México. <http://lef.colmex. $\mathrm{mx} /$ index.php/investigaciones/corpus-sociolingueistico-de-la-ciudad-demexico-cscm $>$.

— y - (coords.) (2015): Corpus sociolingüistico de la ciudad de México, vol. III: Hablantes de instrucción baja, México: El Colegio de México. <http://lef.colmex. $\mathrm{mx} /$ index.php/investigaciones/corpus-sociolingueistico-de-la-ciudad-demexico-cscm $>$.

Martín ZorraQuino, María Antonia y José Portolés (1999): «Los marcadores del discurso». En Ignacio Bosque y Violeta Demonte (eds.), Gramática descriptiva de la lengua española III, Madrid: Espasa Calpe, 4051-4213.

MarTínez, Hernán y Carmen Domínguez (2006): «Análisis prosódico de algunos marcadores discursivos en el habla de Mérida, Venezuela», Lingüistica española actual 28/2, 247-264.

MendozA, Érika y Josaphat GuILLÉn (2016): «Sobre la imbricación de las funciones de la entonación y marcadores discursivos en la afasia de Wernicke», Estudios de Lingüistica Aplicada 63/1, 133-152 (DOI: 10.22201/enallt.01852647p. 2016.63.628). 
MuÑoz, María (2017): «Análisis descriptivo de los valores del marcador discursivo pues en el registro coloquial: aportación de los repertorios lexicográficos», AnMal Electrónica 42, 157-178.

Pascual, Consuelo (2019): «Análisis del marcador discursivo "pues" en las conversaciones en español entre hablantes nativos y hablantes no nativos italianos». En Rosabel Roig-Vila (ed.), Investigación e innovación en la Enseñanza Superior, Barcelona: Octaedro, 1247-1259.

Pons, Salvador (2000): «Los conectores». En Antonio Briz (ed.), ¿̨ Cómo se comenta un texto coloquial?, Barcelona: Ariel, 193-220.

- (2003): «From Agreement to Stressing and Hedging: Spanish bueno and claro». En Gudrun Bachleitner-Held (ed.), Partikeln und Höfflichkeit, Berna: Peter Lang, 219-236.

- (2007): Reseña de Catherine E. Travis, Discourse Markers in Colombian Spanish. A Study in Polysemy, en Spanish in Context 4/2, 303-312 (DOI: 10.1075/sic.4.2. 11pon).

- (2008): «La combinación de marcadores del discurso en la conversación coloquial: interacciones entre posición y función», Estudos Linguísticos/Linguistics Studies 2, 141-159.

- (2016): «Cómo dividir una conversación en actos y subactos». En Antonio Miguel Bañón et al. (eds.), Oralidad y análisis del discurso. Homenaje a Luis Cortés Rodríguez, Almería: Editorial Universidad de Almería, 545-566.

Porroche Ballesteros, Margarita (2008): «Pues». En Diccionario de partículas discursivas del español (DPDE). <www.dpde.es>.

PorTOLÉs, José (1989): «El conector argumentativo pues», Dicenda 8, 117-133.

- (1998): Marcadores del discurso, Barcelona: Ariel.

Roulet, Eddy, Antoine Auchlin, Jacques Moeschler y Christian Rubattel (1985): L'articulation du discours en français contemporain, Berna: Peter Lang.

—, Marcel Burger, Laurent Filliettaz y Anne Grobet (2001): Un modèle et un instrument d'analyse de l'organisation du discours, Berna: Peter Lang.

Travis, Catherine (2005): Discourse Markers in Colombian Spanish: A Study in Polysemy, Berlin-New York: Mouton de Gruyter.

URIBE Mallarino, María del Rosario (2005): «Conectores y contrastividad: el caso de pues». En Lorenzo Blini et al. (eds.), Lingüistica contrastiva entre el italiano y las lenguas ibéricas, vol. II, Roma-Madrid: Instituto Cervantes, 563-578.

- (2006): Dos estudios sobre el conector pues, Milán: LED.

VÁzQuez, Ariel (2013): «Responding and Clarifying: An Analysis of pues as a Sequential Marker in Mexican Spanish Talk-in-interactions», Spanish in Context 10/2, 284-309 (DOI: 10.1075/sic.10.2.05vaz).

- (2017): “"Chilapa pues": variación regional en el uso de pues en posición final en el español mexicano», XVII Congreso Internacional ALFAL, Bogotá: Universidad Nacional.

VILLA, Gisela (2017): Usos y funciones del marcador discursivo pues en el habla limeña. Un caso de difusión contrajerárquica, Tesis de maestría, Lima: Pontificia Universidad Católica del Perú. 